\title{
Pre-Assessment of Radiated Fields from Small Electronic Submodules
}

\author{
Tomas KORINEK, Martin VALEK, Jan SPACIL, Milos MAZANEK \\ Dept. of Electromagnetic Field, Czech Technical University in Prague, Technicka 2, 16627 Praha, Czech Republic
}

\{tomas.korinek, mazanekm, jan.spacil\}@ fel.cvut.cz, martin_valek@yahoo.com

Submitted January 1, 2018 / Accepted June 15, 2018

\begin{abstract}
A novel approach to the evaluation of electromagnetic field radiated from electronic devices by using a small shielded enclosure is presented. Several EMC problems can be identified by employing the computations of a Radiated Model device, such as the model of the Submodule-on-Motherboard structure described here. The combination of a numerical approach, through a validation model and measurements, must be done during the design phase in multiple iterative loops to minimize the risk of noncompliance of the final qualification process. This novel combined approach requires little investment and is highly efficient for industrial applications.
\end{abstract}

\section{Keywords}

Electromagnetic compatibility (EMC), electromagnetic interference (EMI), radiated model, measurement, radiated emissions

\section{Introduction}

Electromagnetic compatibility (EMC) compliance measurements using CISPR 22 [1] entail significant cost and time demands which may be lessened by using precompliance and pre-assessment measurements to characterize the equipment during their design and development. As professional EMC pre-compliance measurement equipment and accessories are too expensive for many small R\&D companies, the use of "In-house" measurement equipment and accessories has become popular.

This paper analyzes the usage of small shielded enclosures for pre-compliance and pre-assessment measurements of electromagnetic field emissions. The combined approach is used to discover worst-case scenarios of EM-field emissions, as demonstrated using the example of a Submoduleon-Motherboard structure (Fig. 1).

According to CISPR 22 [1], the most critical frequency range and peak value of an electric field emitted from a submodule are identified by the analytical solutions of a radiation model (Fig. 2) in combination with emission measurements using a small shielded enclosure and a semi-anechoic chamber. The correlation of these three approaches demonstrates the possibility of utilizing a small shielded enclosure with a suitable receiving antenna for pre-compliance measurements of radiated emissions.

\section{Radiation Model}

To analyze the electromagnetic fields radiated from submodules, selected signals routed between the submodule and motherboard were used. This is typically performed through an inter-board connector while the return-signal path is provided by ground pins. A simplified horizontal Submodule-on-Motherboard structure with the signal pin and ground pins is shown in Fig. 1. The dimensions of the selected motherboard are $230 \mathrm{~mm} \times 220 \mathrm{~mm}$ and for the horizontal submodule $70 \mathrm{~mm} \times 50 \mathrm{~mm}$.

The radiation model has two main parts: A) a Connector Equipment Circuit; and B) an Antenna Model (Fig. 2). The radiation model uses a circuit, which is the equivalent of the inter-board connector, in combination with an antenna model of the Submodule-on-Motherboard structure. The situation is illustrated by the lumped-element model [3] in Fig. 2. There is a potential difference $V_{\text {ant }}$ between the reference planes of the motherboard and the submodule. $V_{\text {ant }}$ originates from a small, but non-negligible signal-return impedance in the inter-board connector provided by the ground pins [2]. $Z_{\mathrm{t}}$ denotes the total signal impedance. The Submodule-on-Motherboard structure acts as a monopole antenna supplied by the voltage source $V_{\text {ant }}$.

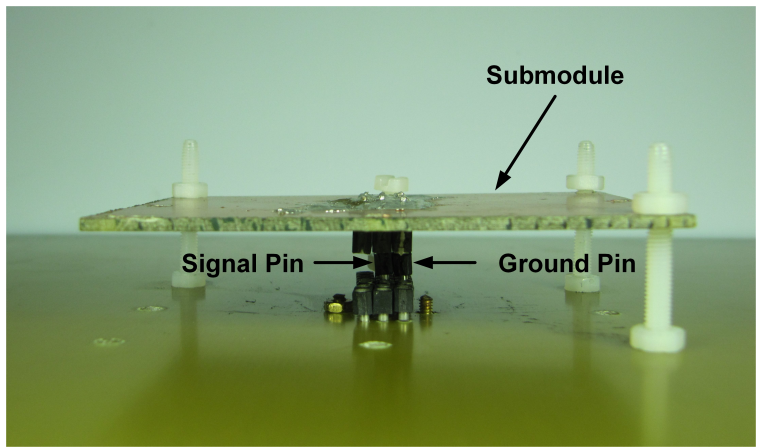

Fig. 1. Submodule-on-Motherboard structure 


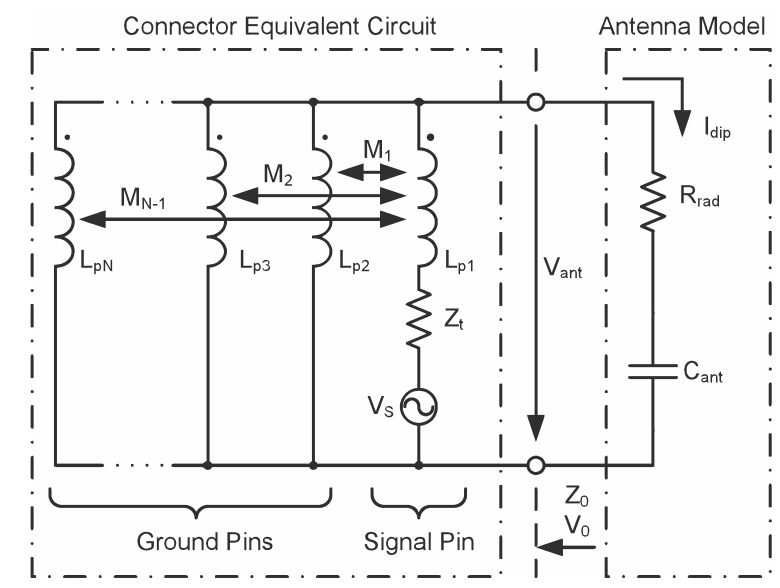

Fig. 2. The lumped-element model of a small horizontal submodule [3]

\subsection{Connector Equivalent Circuit}

The self-partial inductance $L_{p}$ of a pin with a circular cross section can be computed from [4] as:

$$
L_{p}=\frac{\mu_{0}}{2 \pi} h\left[\ln \left(\frac{2 h}{\rho}+\sqrt{\left(\frac{2 h}{\rho}\right)^{2}+1}\right)+\frac{\rho}{2 h}-\sqrt{\left(\frac{\rho}{2 h}\right)^{2}+1}\right]
$$

when the physical dimensions are known. Pin radius is $\rho$, the connector height $h(\rho=0.4 \mathrm{~mm}$ and $h=15 \mathrm{~mm}$ for the structure in Fig. 1), and $\mu_{0}$ represents the permeability of the vacuum. Each pin is coupled with its neighbor by a mutual partial inductance $M_{n}$ :

$$
M_{n}=\frac{\mu_{0}}{2 \pi} h\left[\ln \left(\frac{2 h}{n s}+\sqrt{\left(\frac{2 h}{n s}\right)^{2}+1}\right)+\frac{n s}{2 h}-\sqrt{\left(\frac{n s}{2 h}\right)^{2}+1}\right]
$$

where $n=1,2,3 \ldots(N-1)$ and $N$ are integer indices (number of pins) and $s$ is the uniform pin separation given by the connector pitch. The mutual partial inductance (2) can be alternatively expressed by the coupling factor:

$$
K_{n}=\frac{M_{n}}{L_{p}} .
$$

The mutual inductance $M_{n}$ decreases with increasing multiple distance $n s$. Thus, for the coupling factor $K$ applies:

$$
K_{1}>K_{2}>K_{3} \cdots>K_{N-1}
$$

\subsection{Antenna Model}

The Submodule-on-Motherboard structure behaves as a natural radiator of electromagnetic fields, i.e. an antenna. The radiation pattern, described in [5], of the horizontal Submodule-on-Motherboard structure is similar to the far field of the Hertzian dipole (in this case a Hertzian monopole). The structure radiation parameters were simulated using Method of Moments (MoM) and compared with the measurement in an anechoic chamber. This simplification is correct for the frequency above the first resonance.

The resonance frequency of the structure used is determined by the LC components combination (explained below). The inductance is based on the inductive character of the inter-board connector pins described by the Connector Equivalent Circuit (Fig. 2) in the previous section. The capacitance $C_{\text {ant }}$ between the boards is given by submodule geometry and its value is different for horizontal and vertical Submodule-on-Motherboard structures [6]. The parameter $C_{\text {ant }}$ represents, together with the radiation resistance which is shown as $R_{\text {rad }}$ in Fig. 2 and is critical to radiation amplitude at the resonance, an antenna equivalent circuit known as the Antenna Model. According to results presented in [5], the antenna should be modeled as a Hertzian element. While the submodule is usually much smaller than the motherboard, $C_{\text {ant }}$ can be considered as the capacitance of a metallic patch above a ground plane. The effective area of the submodule is larger than the physical one due to fringing effects and the reference plane to $h / 2$ [7] is used to account for the fringing fields. By applying the image principle we obtain:

$$
C_{\mathrm{ant}}=\varepsilon_{\mathrm{r}} \varepsilon_{0} \frac{(l+2 h)(w+2 h)}{h}
$$

where $l(l=70 \mathrm{~mm})$ and $w(w=50 \mathrm{~mm})$ are the dimensions of the submodule board and $\varepsilon_{\mathrm{r}}$ and $\varepsilon_{0}$ are the relative permittivity and permittivity of the vacuum.

Radiation resistance $R_{\text {rad }}$ is given by half of the radiation resistance of a Hertzian dipole [8] according to the image principle:

$$
R_{\mathrm{rad}}=\frac{1}{2} 80 \pi^{2}\left(\frac{2 h}{c_{0}} f\right)^{2} .
$$

The resonance in the lumped-element equivalent-circuit representation of the structure (Fig. 2) occurs by combining the capacitance $C_{\text {ant }}$ and the total inductance $L_{\text {tot }}$. The resonant frequency $f_{\text {res }}$ can be determined as:

$$
f_{\mathrm{res}}=\frac{1}{2 \pi \sqrt{L_{\mathrm{tot}} C_{\mathrm{ant}}}} .
$$

The total inductance $L_{\text {tot }}$ (for $N_{\mathrm{G}}>1$ ) can then be roughly estimated [3]:

$$
L_{\mathrm{tot}} \approx L_{p} \frac{1+K_{1}}{4}\left(\frac{1}{1+\frac{\left(N_{\mathrm{G}}-2\right)\left(1+K_{1}\right)}{2\left(1+2 K_{1}\right)}}+\frac{1}{1+\frac{\left(N_{\mathrm{G}}-1\right)\left(1+K_{1}\right)}{2\left(1+2 K_{1}\right)}}\right)
$$

where $N_{\mathrm{G}}$ is the total number of grounded pins.

In the case of $N_{\mathrm{G}}=1$ (only one ground pin) we can roughly estimate:

$$
L_{\mathrm{tot}} \approx L_{p} \frac{1}{2}\left(1+\frac{1+K_{1}}{2}\right) .
$$

Approximations (7) and (8) are based on the omission of all mutual couplings with $n>1$. 
For the worst-case estimation, only maximum electric field strength $E_{\max }$ in the far field is considered. Factor 2 in (9) accounts for the infinite ground plane (motherboard). Hence, $E_{\max }$ of a Hertzian source [8], is:

$$
E_{\max }=2 \frac{\eta_{0} \beta I_{\mathrm{dip}} h}{4 \pi r}
$$

where $\eta_{0}=120 \pi$ is the free space wave impedance, $\beta$ is the phase constant and $r$ is the observation distance. $I_{\text {dip }}$ represents the antenna current flowing through $C_{\text {ant }}$, as shown in Fig. 2. The dipole current $I_{\text {dip }}$ given by the antenna voltage $V_{\text {ant }}$ is:

$$
I_{\mathrm{dip}}=\frac{\omega C_{\mathrm{ant}} V_{\mathrm{ant}}}{\sqrt{\left(\omega C_{\mathrm{ant}} R_{\mathrm{rad}}\right)^{2}+1}} \approx \omega C_{\mathrm{ant}} V_{\mathrm{ant}} .
$$

The amplitude of the radiated electric field is expressed:

$$
E_{\max }=\frac{\mu_{0} \omega^{2} C_{\mathrm{ant}} V_{\mathrm{ant}} h}{2 \pi r} .
$$

\section{Measurements in the Shielded Enclosure}

Shielding enclosures protect the equipment against EM disturbances from the environment outside the enclosure, or, to provide a shield to radiated EM fields from sources inside the enclosure.

\subsection{Shielded Enclosure}

A shielded enclosure with inner walls fully covered with microwave absorbers measures radiated emissions from a small submodule whose dimensions are reasonably smaller in comparison to the wavelength (typically $<\lambda / 10$ ).

The residual reflections contribute to the direct path wave (Fig. 3) causing a fading effect [9] which increases the measurement's uncertainty. However, the reflections, as well as the cable loss and connector reflections, can be partly compensated for by the calibration [11].

The physical dimensions of the enclosure (in our case $600 \mathrm{~mm} \times 700 \mathrm{~mm} \times 1000 \mathrm{~mm}$ as seen in Fig. 3) are determined by the need for a resonance distance between the equipment being tested and the top of the absorbers inside the enclosure. Dimensions determine the resonance frequencies of the metallic enclosure $f_{\mathrm{r}_{-} \text {enclosure }}(12)$ (see Fig. 5). The enclosure dimensions should be derived from any rectangular shape e.g., a communication rack.

$$
f_{\mathrm{r}_{-} \text {enclosure }}=\frac{c_{0}}{2 \pi} \sqrt{\left(\frac{m \pi}{a}\right)^{2}+\left(\frac{n \pi}{b}\right)^{2}+\left(\frac{p \pi}{c}\right)^{2}}
$$

where $a, b$ and $c$ are the dimensions of the enclosure and $m, n, p=0,1,3,4, \ldots$ which are mode-order numbers.

\subsection{Measuring Antenna}

For radiation detection from submodules, planar spiral antennas are often used. The spiral antenna is a selfcomplementary structure that has a frequency independent input impedance close to the theoretical value of $60 \pi$, however, in a real environment lower impedance is usually achieved. The spiral antenna consists of two identical arms which are shifted by $180^{\circ}$ with respect to each other. The shape of the spiral arms can be seen as the filling of two identical curves shifted by $90^{\circ}$.

A self-complementary spiral antenna, with outer dimensions of $400 \mathrm{~mm} \times 400 \mathrm{~mm}$ terminating into a short with coaxial feeding, was designed [10] to perform the field measurement and can be seen in Fig. 3. FR-4 substrate of $1 \mathrm{~mm}$ thickness with a relative permittivity of $\varepsilon_{\mathrm{r}}=4.2$ was used. More details can be found in [11].

The designed self-complementary spiral antenna is a circular polarized antenna. The gain of the antenna is measured in the enclosure by a method involving two identical antennas (Fig. 3).

The antenna parameters, such as input impedance and gain, are strongly affected by the environment where the antenna is placed. To calculate the antenna factor $A F_{\mathrm{r}}$ accurately it is necessary to know the correct values of the antenna gain inside the enclosure. Antenna gain and antenna factor are valid for geometry configuration in Fig. 3 and Fig. 5. Finite reflection of the absorbers placed in the antenna near-field region causes uncertainties, which are included in the antenna gain and antenna factor. The condition of the minimum radiated near-field zone is necessary.

The measurement setup (connection of the vector network analyzer, coaxial cables), as well as the distance between the planar spiral antennas, was the same for the gain and the radiated field measurements. Antenna gain $G_{\mathrm{r}}$ was computed from the voltage transmission $s_{21}$ values and its measured frequency dependence is depicted in Fig. 4. The gain indicates that the bottom limit $-3 \mathrm{dBi}$ (see Fig. 4) in the frequency domain is $261.6 \mathrm{MHz}$. Consequently, below this frequency antenna efficiency is too low and the minimum dimensions of the direct path are not fulfilled.

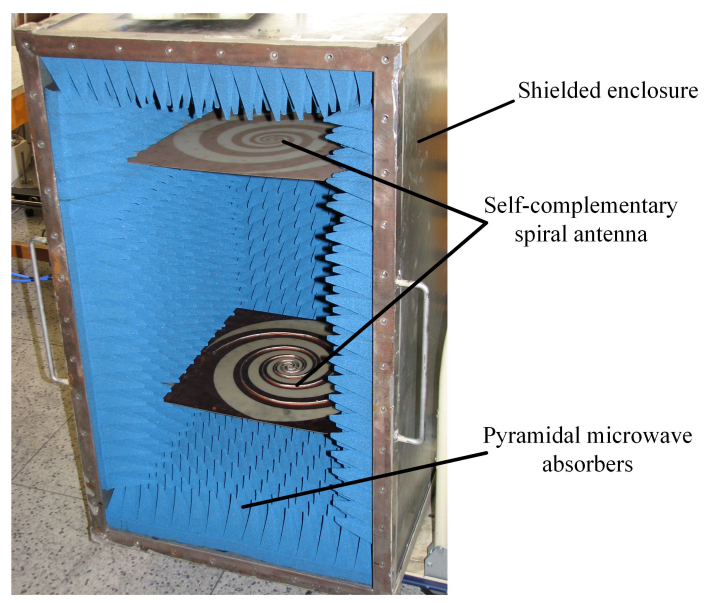

Fig. 3. Measurement configuration for gain and calibration. 


\subsection{Measurement Setup and Results from Submodule Radiation}

A magnitude of electric field emitted from a Submodule-on-Motherboard structure was measured within a shielded enclosure the size of a 19" communication rack $(600 \mathrm{~mm} \times 700 \mathrm{~mm} \times 1000 \mathrm{~mm})$. The interior of the enclosure was covered with pyramidal polyamide-carbon absorbers APM12 and the front wall of the enclosure was removable. Flat microwave absorbers for covering the inner walls of the enclosure can be used too. The function of the absorbers in this case is to decrease the enclosure quality factor, not to simulate full anechoic chamber.

The distance $d$ (see Fig. 5) between the submodule and the receiver antenna in the shielded enclosure was $0.6 \mathrm{~m}$. The submodule position corresponds to the bottom antenna (see Fig. 3). The results $\left(E_{\max }\right)$ consist of maximum value measured from the three submodules positions (see Fig. 5) in coordinating system at the same frequency points.

The Submodule-on-Motherboard structure was fed by a tracking generator at port \#1 (transmitter) of the vector analyzer Agilent E8364A while a voltage on the planar spiral antenna output was evaluated by a built-in detector at port \#2 (receiver) of the same vector analyzer which operated in transmission mode whereby the tracking generator was synchronized with the receiver. In order to compensate for cable losses and connector reflections, the transmission path between the transmitter and the receiver was calibrated. The input power at the $50 \Omega$ SMA connector of the Submodule-on-Motherboard structure was $20 \mathrm{dBmW}$. The measurement set-up is schematically shown in Fig. 5.

The transmission path was calibrated using the standard Open-Short-Match-Through calibration method. The gain $G_{\mathrm{r}}$ of the planar spiral antenna was determined from the measurements of parameter $s_{21}$ which represents a transmission between the transmitter (bottom spiral antenna) and the receiver (top spiral antenna), as you can see in Fig. 3. In the second step, the voltage at the planar spiral antenna $V_{\text {ant }}$ was determined from the measurement of parameter $s_{21}$ which represents a transmission between the transmitter (submodule-on-motherboard placed in the enclosure) and the received antenna (top spiral antenna), as you can see in Fig. 5. The mutual relationship between the incident field and the voltage at the antenna connector is:

$$
E_{\max }[\mathrm{dB} \mu \mathrm{V} / \mathrm{m}]=V_{\text {ant }}[\mathrm{dB} \mu \mathrm{V}]+A F_{\mathrm{r}}[\mathrm{dB} / \mathrm{m}] .
$$

The antenna factor $A F_{\mathrm{r}}(14)$ is a function of frequency and the planar spiral antenna gain $G_{\mathrm{r}}$ :

$$
A F_{\mathrm{r}}[\mathrm{dB} / \mathrm{m}]=20 \log f[\mathrm{MHz}]-29.8-G_{\mathrm{r}}[\mathrm{dBi}] .
$$

The electric field magnitude $E_{\max }[\mathrm{dB} \mu \mathrm{V} / \mathrm{m}]$ measured within the shielded enclosure was recomputed from $0.6 \mathrm{~m}$ distance $d$ to $3 \mathrm{~m}$ distance $D$ in the free space by adding a free space loss $L$ :

$$
L=20 \log (D / d)[\mathrm{dB}] .
$$

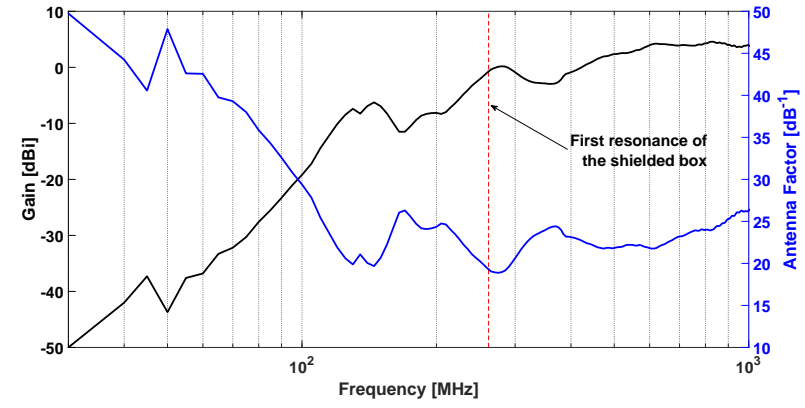

Fig. 4. Gain of the spiral antenna as measured in the shielded enclosure.

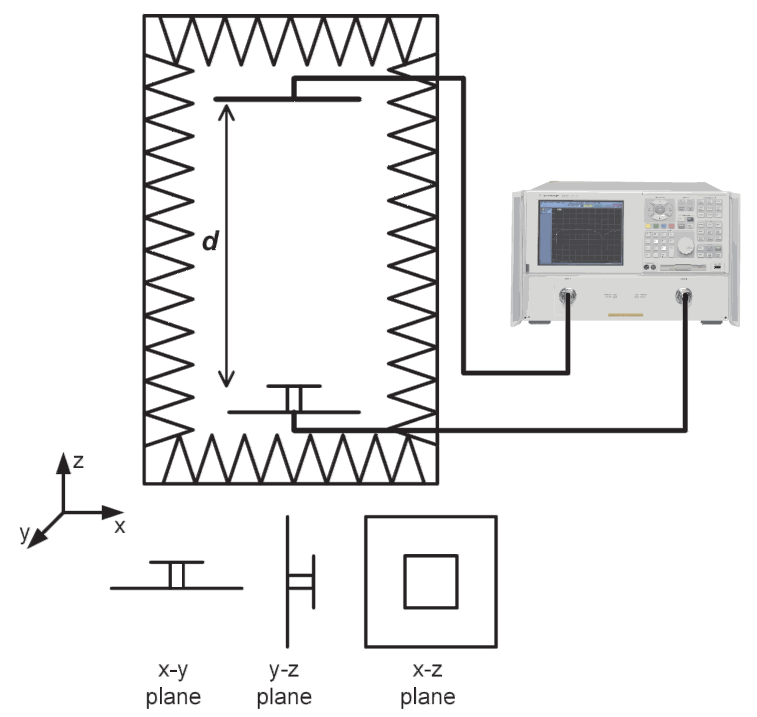

Fig. 5. Radiated electric field measurement in a shielded enclosure, measurement setup.

We suppose that the Submodule-on-Motherboard structure is a source of a linear polarized wave [5]. Once a linear polarized wave is received by a spiral antenna, a polarization loss $L_{\text {pol }}$ of $3 \mathrm{~dB}$ at the antenna should be added.

The environment within the shielded enclosure is similar to the situation in a semi-anechoic chamber where only a direct path wave occurs. Reflections from the ground contribute to the total incident field at the antenna while being measured in a semi-anechoic chamber (SAC). To translate the experimental results presented in this paper to SAC level, a correction factor LFS-to-OATS (FS stands for Free Space and OATS stands for Open Area Test Site) is applied resulting in an approximate value of $5 \mathrm{~dB}$ [12]. This correction was verified in GTEM cell. $L_{\text {total }}$ comprises all losses and factors mentioned above, thus:

$$
L_{\text {total }}[\mathrm{dB}]=L[\mathrm{~dB}]+L_{\mathrm{pol}}[\mathrm{dB}]-L_{\mathrm{FS} \text {-to-OATS }}[\mathrm{dB}] \text {. }
$$

The final expression for the computation of electric field magnitude $E_{\max }[\mathrm{dB} \mu \mathrm{V} / \mathrm{m}]$ in the shielded enclosure, scaled to OATS levels at a distance of three meters, is presented in Fig. 6 (dash-dotted line). The expression for $E_{\max }$ is as follows:

$$
E_{\max }[\mathrm{dB} \mu \mathrm{V} / \mathrm{m}]=V_{\mathrm{ant}}[\mathrm{dB} \mu \mathrm{V}]+A R_{\mathrm{r}}[\mathrm{dB} / \mathrm{m}]-L_{\mathrm{total}}[\mathrm{dB}] .
$$




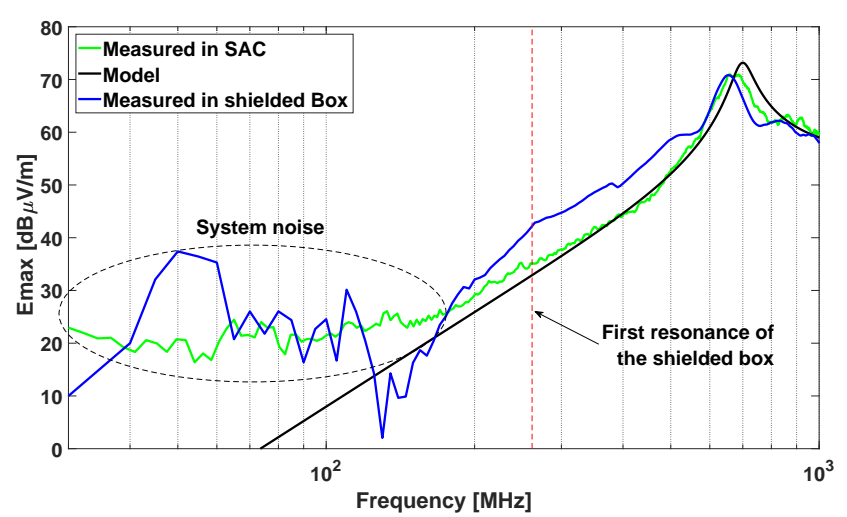

Fig. 6. Magnitude of radiated electric field at a distance of 3 meters.

The computational results using the radiation model and the field measurements in the shielded enclosure are compared with CISPR 22 compliancy measurements in a semi-anechoic chamber. Measured results from the semianechoic chamber, the shielded enclosure, and results calculated from radiated emissions were scaled to a distance of three meters. A comparison of the final measured and calculated dependences is shown in Fig. 6. The same Submodule-on-Motherboard structure was used for both computations through the radiation model and the measurement in the shielded enclosure.

The planar spiral antenna itself and the total setup do not meet the necessary conditions when the first resonant frequency is below $261.6 \mathrm{MHz}$, though the overall maximum field strength at $670 \mathrm{MHz}$ has been identified by both approaches: the shielded enclosure measurement and the radiation model. The combined approach (a simple measurement in a small enclosure together with a numerical model) is recommended to avoid the situation where the final test of the product does not fulfill the level of EMI given by CISPR 22 .

\section{Advantages and Disadvantages of Measurement in a Shielded Enclosure}

Low purchase cost, easy handling and short preparation time for measurement setup make the shielded enclosure attractive for qualification pre-assessments. A wideband planar spiral antenna can further leverage the advantages of obtaining measurements in a shielded enclosure.

The results have proven to be repeatable and variations over several measurement trials meet expectations for engineering applications, though the pre-assessment of radiated emissions in the shielded enclosure does suffer from the limited space available for equipment being tested. In addition, the field measured in lower frequency bands falls into the antenna near-field radiating zone where their validity is limited.
The ultimate limitation of this method is given by the self-resonance of the enclosure: below this frequency, measurement uncertainty increases (see Fig. 6). Electric field measurement is also strongly affected by the electrical parameters (return loss and gain) of the receiving antenna. It is obvious (from Fig. 6) that the measurement error obtains a value of $8 \mathrm{~dB}$ in the frequency band from the first resonance of the enclosure up to $550 \mathrm{MHz}$. This part of the band (see Fig. 6) correlates with the shape of the antenna gain (see Fig. 4) because the antenna gain values are too low in this frequency band.

The antenna electrical parameters (low values of the gain and high values of the antenna factor at low frequencies) cause an increasing the system noise level in the underresonant frequency band (Fig. 6).

\section{Conclusions}

The advantages of the combined approach to a radiated field evaluation include a high level of confidence in the delivered results, low initial investments and exceptional efficiency in terms of time. The positive impact of the EMC measurement, which was introduced into the design of printed circuit boards with submodules, can be investigated in numerous iterative design loops. It is recommended to use a combination of a modeling approach, such as the radiation model used in the pre-assessment and experimental measurements in the shielded enclosure to avoid situations in which critical EMC events appear beyond the applicable frequency range of the planar spiral antenna.

The combined approach creates a fast and cost-effective way to obtain a pre-assessment of radiated electric fields for design engineers.

The "small" shielded enclosure is also suitable for under-resonant region measurements. Semi-anechoic chambers are also often used in under-resonant region. Underresonant region influences are included in the correction factor (antenna factor).

Even if field measurements in a shielded enclosure have limited accuracy, and the radiation model assumes significant simplifications of the inter-board connector, the combination of the two approaches attains a level of accuracy sufficient for engineering purposes.

One of the possible method for decreasing uncertainties is using a known reference noise source as device under test to determine correct antenna factor (calibration) for the geometry of configuration.

The final measurement uncertainty is a sum of partial uncertainties in the measurement setup. Compare with typical measurement setup in an anechoic or semi-anechoic chamber we can, in this case, find an additional uncertainties. 
- Determination of the antenna factor from the antenna gain measurement using the two identical antennas.

- Changing of the measurement distance for different configuration of the DUT in the coordinating system.

- Imperfection of the measurement site, such as normalization site attenuation (NSA). In our case, NSA values are higher than the values in full or semi-anechoic chamber.

According to partial uncertainties, the final uncertainty of the measurement in the "small" shielded enclosure will be higher than the measurement uncertainty for full anechoic chamber [13] and for semi-anechoic chamber [13].

\section{References}

[1] CISPR 22: 2003-04. Information Technology, Equipment - Radio Disturbance Characteristics - Limits and Methods of Measurement.

[2] LI, K., TASSOUDIJ, A., POH, S. Y., et al. FD-TD analysis of electromagnetic radiation from modules-on-backplane configurations. IEEE Transactions on Electromagnetic Compatibility, 1995, vol. 37, no. 3, p. 326-332. DOI: $10.1109 / 15.406522$

[3] VALEK, M., LEONE, M. Estimation of radiated fields of small horizontal submodules based on a lumped-element model. Radioengineering, 2006, vol. 15 , no. 4, p. 9-15.

[4] PAUL, C. R. Introduction to Electromagnetic Compatibility. New York: John Wiley \& Sons, 1992. ISBN: 978-0-471-75500-5

[5] VALEK, M., LEONE, M., SCHMIEDL, F. Analysis of the radiation behaviour of motherboard-subboard structures. In Proceedings of the 6th International Symposium on Electromagnetic Compatibility and Electromagnetic Ecology. St. Petersburg (Russia), 2005, p. 175-178. DOI: 10.1109/EMCECO.2005.1513094

[6] LEONE, M., NAVRATIL, V. On the electromagnetic radiation of printed-circuit-board interconnections. IEEE Transactions on Electromagnetic Compatibility, 2005, vol. 47, no. 2, p. 219-226. DOI: 10.1109/TEMC.2005.847400

[7] LANGE, K., LOCHERER, K. H. Taschenbuch der Hochfrequenztechnik. Springer-Verlag, 1986. ISBN: 978-3540547143

[8] BALANIS, C. A. Antenna Theory. New York: John Wiley \& Sons, 1997. ISBN: 978-0471667827

[9] RAPPAPORT, T. S. Wireless Communications - Principles and Practice, 2nd. ed., Prantice Hall, 2002. ISBN: 978-0130422323

[10] PIKSA, P., MAZANEK, M. A self-complementary 1.2 to $40 \mathrm{GHz}$ spiral antenna with impedance matching. Radioengineering, 2006, vol. 15 , no. 3 , p. $15-19$.

[11] KORINEK, T., PIKSA, P., MAZANEK, M. Wideband measurement in a small shielded box using equiangular spiral antennas. Radioengineering, 2006, vol. 15, no. 4, p. 34-37.
[12] VALEK, M., LEONE, M. Radiated emission measurement of printed circuit boards in the GTEM cell and GTEM-to-OATS correlation factor. In Proceedings of the 13th Conference on Microwave Techniques (COMITE). Prague (Czech Republic), 2005, p. 155-158.

[13] CSN EN 55016-4-2. Specification for Radio Disturbance and Immunity Measuring Apparatus and Methods. Uncertainties, Statistics and Limit Modelling. Measurement Instrumentation Uncertainty

\section{About the Authors...}

Tomas KORINEK was born in Jicin, Czech Republic, in 1979. He received his M.Sc. and Ph.D. degree in Radioelectronics from the Czech Technical University in Prague (CTU) in 2005, resp. 2012. From 2007 to 2008, he was a research and designer engineer at RFspin s.r.o., where he was engaged in antennas and microwave circuits. Currently he is an Assistant Professor and the head of the Laboratory at the Department of Electromagnetic Field of the Czech Technical University in Prague. His research interests include the area of EMC and antennas.

Martin VALEK was born in Prague, Czech Republic, in 1978. He received his M.Sc. and Ph.D. degree in Radioelectronics from the Czech Technical University (CTU) in Prague in 2003, resp 2011. He worked as an EMC engineer for the automotive industry from 2003 to 2004. Between 2004 and 2006, he worked for Siemens Communications where he started with the research of the EMI from submodules in the Center of Quality Engineering in Munich, Germany. In 2006, he joined Siemens VDO as a project manager. After several years of leading NPI projects he joined General Electric (GE) in 2010. He works as a commercial manager for the CEE region for GE Energy Services in Prague (Czech Republic).

Jan SPACIL was born in Brandys nad Labem, Czech Republic, in 1990. He received his M.Sc. degree in Communication Technology from the Czech Technical University (CTU) in Prague in 2016. He is currently working toward the Ph.D. degree at the Czech Technical University in Prague. His research interests include the area of EMC and microwave measurements.

Milos MAZANEK was born in Tanvald, Czech Republic, in 1950. He received his M.Sc. and Ph.D. from the Czech Technical University in Prague (CTU) in 1974, resp. 1980. Since the year 2000 he has been a full professor at CTU in Prague. In the period 1997-2014 he was a head of the Department of Electromagnetic Field. His professional work is focused on Antennas, EMC, Microwave Radiometry and Remote Sensing and Propagation aspects. He was a chair of the EuCAP (European conference on Antennas and Propagation) conference 2012 held in Prague. 